\title{
PREDICCIÓN DE VALORES GENÉTICOS DEL EFECTO DE POBLACIONES DE MAÍZ EVALUADAS EN BRASIL Y PARAGUAY
}

\author{
Predicting breeding values of population effect of maize evaluated \\ in Brazil and Paraguay
}

\author{
Freddy Mora ${ }^{1}$ * y Carlos Alberto Scapim ${ }^{1}$
}

\begin{abstract}
A B S T R A C T
The aim of this study was to evaluate the performance of seven popcorn (Zea mays L.) populations of Avati Pichinga landrace, established in different sites of Brazil and Paraguay, determining and analyzing the empirical best linear unbiased predictor (EBLUP) of population effect, and estimating covariance parameters by the restricted maximum likelihood (REML) method, for commercial and agronomic characteristics. Ten models were investigated for fitting an appropriate intergenotype covariance structure. Akaike and Bayesian information criteria were used to compare alternative models. The covariance structures chosen were Toeplitz with three bands, for grain yield; Toeplitz with two bands for both popping expansion and plant height; autoregressive for ear height; and variance component for blooming. Population effect was significant for all the agronomic traits, unlike genotype-environment interaction. Spearman correlation coefficients were not significant between grain yield and popping expansion ranking. P4 and P5 can be considered as promissory populations according to their predicted breeding values for grain yield; P1 and P4 for popping expansion. These populations can be regarded as useful for popcorn breeding programs.
\end{abstract}

Key words: covariance structures, yield, popping expansion, Zea mays L.

\section{R E S U M E N}

El objetivo de este estudio fue evaluar el comportamiento de siete poblaciones de maíz ( $Z e a$ mays L.) tipo roseta, o palomita, de la raza Avati Pichinga, establecidas en diferentes localidades de Brasil y Paraguay. Se determinó la mejor predicción lineal no sesgada empírica (EBLUP), con estimaciones de parámetros de covarianza vía método de máxima verosimilitud restringida (REML), en características de importancia comercial y agronómica. Se investigaron diez modelos con diferentes estructuras de covarianzas en la matriz intergenotípica. Los criterios de información de Akaike y Bayesiano de Schwarz se utilizaron para la comparación entre los modelos. Las estructuras de covarianza escogidas fueron Diagonal de tres bandas para el rendimiento de granos, Diagonal de dos bandas para la capacidad de expansión y la altura de planta, Auto-regresiva de primer orden para la altura de mazorca, y tipo componentes de varianza para la floración masculina. El efecto de poblaciones fue significativo, al contrario de la interacción genotipo-ambiente. Correlaciones de Spearman fueron no significativas entre rendimiento y capacidad de expansión. Las poblaciones P4 y P5 se ven promisorias al considerar su valor genético del rendimiento, y P1 y P4 para capacidad de expansión. Tales poblaciones pueden ser consideradas útiles para el programa de mejoramiento.

Palabras clave: estructuras de covarianzas, rendimiento, capacidad de expansión, Zea mays L.

\footnotetext{
${ }^{1}$ Universidade Estadual de Maringá-Paraná, Departamento de Agronomía, Av. Colombo 5790 Bloco 05, CEP:87020-900. Maringá, Paraná, Brasil. E-mail: morapoblete@gmail.com; cascapim@uem.br *Autor para correspondencia. Recibido: 20 de marzo de $2006 . \quad$ Aceptado: 19 de julio de 2006.
} 


\section{INTRODUCCIÓN}

El maíz (Zea mays L.) es el segundo cereal de mayor producción mundial, superado solamente por el trigo (Triticum aestivum L.). Es producido para consumo humano y también es utilizado como recurso energético en los sistemas de producción pecuarios (Jahn et al., 2000; Rojas y Manríquez, 2001). El maíz tipo rosetas, o palomitas de maíz, con capacidad de reventar a altas temperaturas, representa una pequeña fracción de la producción total, pero con una tendencia de crecimiento continuo tanto de la producción como de la productividad (Quintana, 2002).

En un programa de mejoramiento genético del maíz tipo roseta, se debe tomar en consideración las características agronómicas de interés y los aspectos relacionados con la calidad del producto. Por un lado, al agricultor le interesa obtener una productividad elevada y los demás atributos de una buena población de maíz normal, y por otro, al consumidor le interesa una alta capacidad de expansión, junto con una mejor textura y suavidad (Scapim et al., 2002). Avati Pichinga es una raza indígena de maíz con capacidad para la producción de rosetas, la cual está siendo considerada en programas de investigación, en diversos países de América del Sur (Pflüger y Schlatter, 1996; Quintana, 2002). Esta raza también ha sido indicada para mejorar características como la tolerancia a los agentes causales de la pudrición de mazorcas (Quintana, 2002).

El estudio de la variabilidad fenotípica y genética de poblaciones naturales es de gran importancia para definir los materiales que serán utilizados en programas más específicos de mejoramiento vegetal (García et al., 2002). La evaluación genética en diversas condiciones ambientales, determinada a través del valor genético de los individuos o grupos de genotipos, es fundamental para el éxito de las nuevas siembras con la especie.

La evaluación genética de poblaciones agronómicas puede realizarse en ensayos de campo a través de la metodología de modelos lineales mixtos, desarrollada por Henderson $(1949 ; 1950)$. Tal procedimiento fue denominado como la mejor predicción lineal no sesgada (BLUP) (del inglés: best linear unbiased predictor) para definir la metodología de predicción de valores genéticos. Piepho y Möhring (2006) reportaron la utilidad de los modelos lineales mixtos en la selección de cultivares agronómicos de Brassica napus L., en etapas avanzadas de un programa de mejoramiento genético, demostrando que el método de la máxima verosimilitud restringida (REML) (del inglés: restricted maximum likelihood) es preferible a la máxima verosimilitud (ML), en la estimación de componentes de varianza y que BLUP es preferible al mejor estimador linear no sesgado (BLUE) (del inglés: best linear unbiased estimator).

En términos de análisis genético, los valores genéticos son variables aleatorias no observables que deben ser obtenidas a partir de los valores fenotípicos, que son las características observables de interés. La predicción de valores genéticos depende de las estimaciones de componentes de varianza, los cuales deben ser calculados con la mayor precisión posible. En modelos lineales mixtos, el procedimiento estándar que ha sido preferentemente usado en la estimación de componentes de varianza es el de máxima verosimilitud restringida (Mora et al., 2007). Estudios previos de Reis et al. (2005) y de Mora y Arnhold (2006) indicaron que los métodos de estimación/predicción vía REML/BLUP pueden ser también aplicados en ensayos genéticos de los programas de mejoramiento del maíz.

El presente trabajo tuvo como objetivo evaluar el comportamiento de siete poblaciones de maíz de la raza Avati Pichinga, establecidas en una localidad de Brasil y en cuatro localidades de Paraguay, a través de la mejor predicción lineal no sesgada empírica (EBLUP) del efecto poblacional, con estimaciones de componentes de varianza basados en el método de máxima verosimilitud restringida (REML), en características de importancia comercial y agronómica, considerando diferentes estructuras de covarianzas del efecto intergenotípico.

\section{MATERIALES Y MÉTODOS}

Se establecieron varios ensayos que incluyen poblaciones de maíz de la raza Avati Pichinga, para estudiar la variabilidad de diferentes características agronómicas y dar comienzo a un programa de mejoramiento en Brasil y Paraguay.

\section{Material agronómico y descripción de los ensayos} Siete poblaciones de maíz tipo roseta de la raza Avati Pichinga se colectaron al azar en diferentes departamentos administrativos de Paraguay (Cuadro 1), para el establecimiento de cinco ensayos, 
Cuadro 1. Localización general de las poblaciones de maíz tipo roseta de la raza Avati Pichinga colectadas en Paraguay.

Table 1. General localization of popcorn populations of Avati Pichinga landrace collected in Paraguay.

\begin{tabular}{llcc}
\hline Poblaciones & Departamento & $\begin{array}{c}\text { Latitud } \\
\text { Sur }\end{array}$ & $\begin{array}{c}\text { Longitud } \\
\text { Oeste }\end{array}$ \\
\hline AVP1 (P1) & Itapúa & $27^{\circ} 03^{\prime}$ & $55^{\circ} 33^{\prime}$ \\
AVP2 (P2) & Itapúa & $26^{\circ} 35^{\prime}$ & $55^{\circ} 34^{\prime}$ \\
AVP3 (P3) & Misiones & $26^{\circ} 39^{\prime}$ & $57^{\circ} 56^{\prime}$ \\
AVP4 (P4) & Itapúa & $26^{\circ} 35^{\prime}$ & $55^{\circ} 34^{\prime}$ \\
AVP5 (P5) & San Pedro & $24^{\circ} 06^{\prime}$ & $57^{\circ} 05^{\prime}$ \\
AVP6 (P6) & Caazapá & $26^{\circ} 09^{\prime}$ & $56^{\circ} 24^{\prime}$ \\
AVP7 (P7) & Alto Paraná & $25^{\circ} 31^{\prime}$ & $54^{\circ} 37^{\prime}$ \\
\hline
\end{tabular}

en febrero de 2002. En cada ensayo se usó un diseño de bloques completos al azar, con tres repeticiones. Los sitios de ensayo correspondieron a cuatro localidades del distrito paraguayo denominado $\mathrm{Ca}$ pitán Miranda $\left(27^{\circ} 12^{\prime}\right.$ lat. Sur, $55^{\circ} 48^{\prime}$ long. Oeste), y una localidad perteneciente al Municipio de $\mathrm{Pa}$ lotina $\left(24^{\circ} 12^{\prime}\right.$ lat. Sur; $53^{\circ} 50^{\prime}$ long. Oeste), del estado de Paraná, en Brasil.

\section{Colección de datos y características agronómicas}

Las características agronómicas evaluadas correspondieron a la altura de planta (AP, expresada en $\mathrm{cm}$ ), altura de mazorcas (AE, en $\mathrm{cm}$ ), florecimiento masculino (FLOR, en días), rendimiento de granos (REND, en kg ha ${ }^{-1}$ ) y capacidad de expansión (CE, en $\left.\mathrm{mL} \mathrm{mL}^{-1}\right)$. La capacidad de expansión, o volumen de expansión, se obtuvo a través de la razón entre el volumen de la roseta expandida y la masa de los granos. Para cada parcela se colectó una muestra de $30 \mathrm{~g}$ de granos, proveniente de la parte central de las mazorcas, la cual se preparó en flujo de aire caliente y con 1.200 Watts de potencia considerando una temperatura de $280^{\circ} \mathrm{C}$ durante 2 min y $10 \mathrm{~s}$. El peso de los granos se corrigió para una humedad estándar de $14 \%$ antes del análisis de los resultados, considerando la siguiente fórmula:

$R G=\frac{R(1-U G)}{(1-0,14)}$

donde $\mathrm{RG}$ representa el peso de los granos corregidos para $14 \%$ de humedad; $R$ es el peso de los granos no corregidos para la humedad estándar; UG simboliza la humedad de los granos observada; (1-UG) es el contenido de materia seca.

\section{Predicción de valores genéticos}

Se realizaron análisis de varianzas por sitio para la obtención de los cuadrados medios residuales, a objeto de realizar análisis de homogeneidad de varianzas residuales entre los ambientes, y posibilitar el análisis conjunto. Verificada tal condición, se realizó el análisis de varianza, considerando el siguiente modelo lineal mixto (en forma matricial):

$\mathrm{y}=\mathrm{X} \beta+Z \mu+\varepsilon$

donde $y$ es el vector de observaciones fenotípicas (AP, AE, CE, FLOR o REND); $X$ simboliza la matriz de incidencia de los efectos fijos; $\beta$ es el vector de los efectos fijos debido al sitio; $Z$ simboliza la matriz de incidencia de los efectos aleatorios; $\mu$ es el vector de los efectos aleatorios de poblaciones, bloque dentro de sitio e interacción poblaciónsitio; $\varepsilon$ es el vector aleatorio de residuos.

En el Cuadro 2 se muestra el esquema de análisis de varianza para el análisis conjunto, realizado a través del procedimiento GLM de SAS, con suma de cuadrado tipo III, ajustando los valores de F para los efectos de poblaciones y de sitio.

Los vectores de efectos aleatorios $\mu$ y $\varepsilon$ tienen distribución normal, con media cero, y con matrices de covarianzas $G$, y $R=I \sigma_{e}^{2}$ respectivamente. Matricialmente, se tiene la siguiente expresión para la varianza de los efectos aleatorios:

$\operatorname{Var}\left[\begin{array}{l}\mu \\ \varepsilon\end{array}\right]=\left[\begin{array}{cc}G & \varnothing \\ \varnothing & R\end{array}\right]$

Así, la matriz $\mathrm{G}$ está conformada por las matrices de los efectos aleatorios de poblaciones $\left(G_{p}\right)$, de bloque $\left(\mathrm{G}_{\mathrm{b}}\right)$ y de interacción sitio-población $\left(\mathrm{G}_{\mathrm{w}}\right)$.

Se determinó la predicción de valores genéticos del efecto de poblaciones, vía la mejor predicción lineal no sesgada empírica (EBLUP), cuyos componentes de varianza no son conocidos y sí estimados (Littell et al., 1996), considerando las siguientes ecuaciones de modelos mixtos:

$$
\left[\begin{array}{cc}
X^{\prime} R^{-1} X & X^{\prime} R^{-1} Z \\
Z^{\prime} R^{-1} X & Z^{\prime} R^{-1} Z+G^{-1}
\end{array}\right]\left[\begin{array}{l}
\beta \\
\mu
\end{array}\right]=\left[\begin{array}{c}
X^{\prime} R^{-1} y \\
Z^{\prime} R^{-1} y
\end{array}\right]
$$

Para la obtención de los componentes de varianza, necesarios para la obtención de valores genéticos, se utilizó el procedimiento de la máxima verosimilitud restringida (REML). Para la estimación/predicción vía 
Cuadro 2. Esquema de análisis de varianza para el análisis conjunto.

Table 2. Joint analysis of variance scheme.

\begin{tabular}{llll}
\hline $\begin{array}{l}\text { Fuente de } \\
\text { variación }\end{array}$ & $\begin{array}{c}\text { Cuadrados } \\
\text { medios }\end{array}$ & $\begin{array}{c}\text { Cuadrados medios } \\
\text { esperados }\end{array}$ & Pruebas F \\
\hline Sitio (S) & CMS & $\sigma^{2}+3 \sigma_{S^{*} P}^{2}+7 \sigma_{B I S}^{2}+Q_{S}$ & $F=\frac{(C M S+C M E)}{(C M S x P+C M B)}$ \\
Población (P) & $\mathrm{CMP}$ & $\sigma^{2}+3 \sigma_{S x P}^{2}+15 \sigma_{P}^{2}$ & $F=\frac{(C M P)}{(C M S x P)}$ \\
Bloque/S (B/S) & $\mathrm{CMB}$ & $\sigma^{2}+7 \sigma_{B I S}^{2}$ & \\
S x P & $\mathrm{CMSxP}$ & $\sigma^{2}+3 \sigma_{S^{* P}}^{2}$ & $F=\frac{(C M S x P)}{(C M E)}$ \\
Error & $\mathrm{CME}$ & $\sigma^{2}$ & \\
\hline
\end{tabular}

REML/BLUP, se utilizó el procedimiento MIXED (mixed lineal model) de SAS ${ }^{\circledR} 8.02$ (SAS Institute, 1996).

Luego de la caracterización del modelo general, se implantaron estructuras de covarianzas en la matriz $\mathrm{G}_{\mathrm{p}}$, referido a la matriz intergenotípica, o interpoblacional, de tal forma de determinar la mejor estructura que representa la dependencia o independencia entre las observaciones. Con esto se crearon 10 modelos para cada característica considerada (Cuadro 3). Las matrices de covarianzas de los parámetros estimados se obtuvieron a partir de la inversa de la matriz Hessian (Bard, 1974). Los criterios de información de Akaike (AIC, Akaike, 1974) y Bayesiano de Schwarz (BIC, Schwarz, 1978) se utilizaron para la comparación entre los modelos (Mora et al., 2007).

\section{Relación entre características}

Se calcularon los coeficientes de correlación de Spearman entre los ranking de poblaciones deter- minados vía EBLUP, a través del procedimiento CORR de SAS (Mora y Arnhold, 2006), como una medida de relación entre las características. El estadístico t-Student se utilizó para estimar si cada correlación es significativa.

\section{RESULTADOS Y DISCUSIÓN}

En el análisis conjunto existieron diferencias significativas entre las poblaciones $(p<0,01)$ en todos los caracteres analizados (Cuadro 4). Esta variación entre poblaciones indica una situación providencial a la selección inter-genotípica. En Sudamérica, trabajos realizados por Sawazaki et al. (1986) sobre la evaluación de la capacidad de expansión de seis poblaciones de maíz tipo roseta, mostraron amplias posibilidades de mejoramiento de esa característica con el uso de híbridos.

Los coeficientes de variación mostraron niveles aceptables para la experimentación con este tipo de maíz, variando entre 3 y 14,8\%, correspondientes

Cuadro 3. Modelos de las estructuras de covarianzas de la matriz intergenotípica $\left(G_{p}\right)$.

Table 3. Covariance structures models of intergenotype matrix $\left(G_{p}\right)$.

\begin{tabular}{cllr}
\hline Modelo & Matriz $\mathbf{G}_{\mathbf{p}}$ & Definición & Parámetros $\left(\mathbf{N}^{\mathbf{o}}\right)$ \\
\hline 1 A & VC & Componentes de varianza & 4 \\
$1 \mathrm{~B}$ & CS & Simetría compuesta & 5 \\
1 C & HF & Huynh-Feldt & 11 \\
$1 \mathrm{D}$ & TOEP (1) & Diagonal 1 (toeplitz banded) & 4 \\
$1 \mathrm{E}$ & TOEP (2) & Diagonal 2 & 5 \\
$1 \mathrm{~F}$ & TOEP (3) & Diagonal 3 & 6 \\
$1 \mathrm{G}$ & TOEP (4) & Diagonal 4 & 7 \\
$1 \mathrm{H}$ & AR (1) & Auto-regresiva de orden 1 & 5 \\
1 I & CSH & Simetría compuesta heterogénea & 11 \\
1 J & UN & No estructurada & 31 \\
\hline
\end{tabular}


a las características de florecimiento masculino y rendimiento de granos. Scapim et al. (2002) encontraron coeficientes de variación aproximados de 19 y $13 \%$, para las características de rendimiento de granos y capacidad de expansión, respectivamente, en ensayos de cruzamientos dialélicos con maíz tipo rosetas, establecidos en el sur de Brasil.

Los supuestos de normalidad fueron confirmados por los valores del estadístico $\mathrm{W}$, estadístico de Shapiro-Wilk (Cuadro 4), observado en el análisis de datos interactivo de SAS-Insight, y a través de un análisis gráfico (Sokal y Rohlf, 2003).

Cabe destacar el valor observado en la capacidad de expansión media $(\mathrm{CE}=8,66)$, considerado bajo desde el punto de vista comercial, pero normal si se considera que son poblaciones naturales. Según Miranda et al. (2003) para que una población pueda ser comercializada, debe tener una capacidad de expansión sobre 20, ya que valores bajo ese índice la roseta se presenta muy rígida y con muchos granos sin reventar, siendo una característica de las poblaciones o variedades no mejoradas, cuya CE varía entre 8 y 12. La capacidad de expansión es una característica poligénica y que puede también ser afectada por factores externos, ya sean éstos bióticos o abióticos. El contenido de humedad del grano, daños provocados en el pericarpio y endospermo, son algunos de los factores específicos que pueden afectar tal característica (Dofing et al., 1991).

Para los efectos debido al sitio e interacción sitiopoblación no se evidenciaron diferencias estadísti- cas significativas ( $p>0,05)$, indicando un comportamiento invariable de las poblaciones en los ambientes evaluados. La ausencia de interacción genotipo-ambiente implica que la selección puede ser realizada considerando la respuesta promedio de los genotipos, ya que la clasificación genotípica no varía significativamente en los diferentes sitios (Mora et al., 2007).

Los resultados del procedimiento MIXED de SAS basados en los criterios de información Akaike (AIC) (Akaike, 1974) y Bayesiano de Schwarz (Schwarz, 1978) se muestran en el Cuadro 5, para los modelos creados en cada característica considerada. Los tipos de estructuras de covarianzas no mostrados corresponden a aquellos que presentaron dificultades en la obtención de convergencia REML, basada en el algoritmo de Newton-Raphson. El número de iteraciones de este algoritmo varió entre 3 y 12.

Los modelos mixtos seleccionados de acuerdo a los menores valores de AIC y BIC fueron dependientes de la característica evaluada (Cuadro 5). Las estructuras de covarianzas genotípicas fueron: Diagonal 3 (modelo $1 \mathrm{~F}$, Cuadros 3 y 5), para el rendimiento de granos; Diagonal 2 (modelo $1 \mathrm{E}$ ), para la capacidad de expansión y la altura de planta; estructura Auto-regresiva (modelo $1 \mathrm{H}$ ), para la altura de mazorca; y tipo componentes de varianza (modelo $1 \mathrm{~A}$ ) para la característica floración masculina. Estos resultados indican la relación existente entre las poblaciones. El desconocimiento de las variabilidades presentes entre las observaciones

Cuadro 4. Cuadrados medios por característica de poblaciones de maíz tipo rosetas. Se incluyen promedios, coeficiente de variación (CV) y estadístico de normalidad Shapiro-Wilk.

Table 4. Means square of popcorn populations per trait, including mean, coefficient of variation $(\mathrm{CV})$ and normally statistic of Shapiro-Wilk.

\begin{tabular}{lcccccc}
\hline \multirow{2}{*}{$\begin{array}{l}\text { Fuente de } \\
\text { variación }\end{array}$} & gl & $\begin{array}{c}\text { FLOR } \\
\text { (días) }\end{array}$ & $\begin{array}{c}\text { AP } \\
(\mathbf{c m})\end{array}$ & $\begin{array}{c}\text { AE } \\
(\mathbf{c m})\end{array}$ & $\begin{array}{c}\text { REND } \\
\left(\mathbf{k g ~ h a}^{-1}\right)\end{array}$ & $\begin{array}{c}\mathbf{C E} \\
\left(\mathbf{m L} \mathbf{~ m L}^{-1} \mathbf{)}\right.\end{array}$ \\
\hline Bloque/Sitio & 10 & 3,276 & 373,646 & 359,839 & 1.001 & 7,425 \\
Sitio (S) & 4 & $2,571 \mathrm{~ns}$ & $0,525 \mathrm{~ns}$ & $9,502 \mathrm{~ns}$ & $11 \mathrm{~ns}$ & $0,391 \mathrm{~ns}$ \\
Población (P) & 6 & $76,622^{*}$ & $2.602,412^{*}$ & $1.088,013^{*}$ & $3.851 .765^{*}$ & $37,235^{*}$ \\
S x P & 24 & $1,249 \mathrm{~ns}$ & $2,262 \mathrm{~ns}$ & $4,156 \mathrm{~ns}$ & $55 \mathrm{~ns}$ & $0,230 \mathrm{~ns}$ \\
Error & 60 & 5,143 & 137,961 & 83,415 & 78.818 & 1,117 \\
Promedio & & 76,3 & 172,175 & 95,75 & $1.892,2$ & 8,66 \\
CV\% & & 3,0 & 6,8 & 9,5 & 14,8 & 12,2 \\
Shapiro-Wilk (W) & & 0,95 & 0,94 & 0,94 & 0,92 & 0,98 \\
\hline
\end{tabular}

*Diferencias significativas $1 \%$ de probabilidad.

ns: no significativa; FLOR: florecimiento masculino; AP: altura de planta; AE: altura de mazorca; REND: rendimiento de granos; CE: capacidad de expansión. 
Cuadro 5. Estructuras de covarianzas, valores de los criterios de información de Akaike (AIC), Bayesiano de Schwarz (BIC) y -2 veces el logaritmo de la máxima verosimilitud restringida (-2rLL), por modelo y característica.

Table 5. Covariance structures, Akaike information criterion (AIC), Schwarz's Bayesian information criterion (BIC) and -2 restricted logarithm of likelihood values $(-2 \mathrm{rLL})$, per model and trait.

\begin{tabular}{|c|c|c|c|c|c|c|}
\hline Característica & Modelos & $\begin{array}{l}\text { Estructura } \\
\text { matriz Gp }\end{array}$ & $-2 r L L$ & AIC & BIC & $\begin{array}{l}\text { Iteraciones } \\
\text { REML }\left(\mathbf{N}^{\circ}\right)\end{array}$ \\
\hline \multirow{6}{*}{ REND } & $1 \mathrm{~A}$ & $\mathrm{VC}$ & $1.407,9$ & $1.411,9$ & $1.411,7$ & 4 \\
\hline & $1 \mathrm{C}$ & $\mathrm{HF}$ & $1.407,9$ & $1.425,9$ & $1.425,4$ & 7 \\
\hline & $1 \mathrm{D}$ & TOEP (1) & $1.407,9$ & $1.411,9$ & $1.411,7$ & 4 \\
\hline & $1 \mathrm{E}$ & TOEP (2) & $1.405,8$ & $1.411,8$ & $1.411,7$ & 4 \\
\hline & $1 \mathrm{~F}^{1}$ & TOEP (3) & $1.403,4$ & $1.411,4$ & $1.411,2$ & 12 \\
\hline & $1 \mathrm{G}$ & $\mathrm{AR}(1)$ & 1.407 & 1.413 & $1.412,8$ & 5 \\
\hline \multirow{7}{*}{$\mathbf{C E}$} & $1 \mathrm{~A}$ & $\mathrm{VC}$ & 328,4 & 334,4 & 334,3 & 3 \\
\hline & $1 \mathrm{~B}$ & $\mathrm{CS}$ & 328,4 & 336,4 & 336,2 & 4 \\
\hline & $1 \mathrm{C}$ & $\mathrm{HF}$ & 328,4 & 348,4 & 347,9 & 8 \\
\hline & $1 \mathrm{D}$ & TOEP (1) & 328,4 & 334,4 & 334,3 & 3 \\
\hline & $1 \mathrm{E}^{1}$ & TOEP (2) & 326,4 & 334,4 & 334,2 & 8 \\
\hline & $1 \mathrm{~F}$ & TOEP (3) & 325,9 & 335,9 & 335,7 & 7 \\
\hline & $1 \mathrm{H}$ & $\mathrm{AR}(1)$ & 328,4 & 336,4 & 336,2 & 4 \\
\hline \multirow{7}{*}{$\mathbf{A P}$} & $1 \mathrm{~A}$ & $\mathrm{VC}$ & 791,6 & 797,6 & 797,4 & 4 \\
\hline & $1 \mathrm{~B}$ & $\mathrm{CS}$ & 791,6 & 799,6 & 799,4 & 4 \\
\hline & $1 \mathrm{C}$ & $\mathrm{HF}$ & 791,6 & 811,6 & 811 & 9 \\
\hline & $1 \mathrm{D}$ & TOEP (1) & 791,6 & 797,6 & 797,4 & 4 \\
\hline & $1 \mathrm{E}^{1}$ & TOEP (2) & 789,4 & 797,4 & 797,2 & 5 \\
\hline & $1 \mathrm{~F}$ & TOEP (3) & 788,5 & 798,5 & 798,3 & 6 \\
\hline & $1 \mathrm{H}$ & AR (1) & 789,9 & 797,9 & 797,7 & 5 \\
\hline \multirow{5}{*}{$\mathbf{A E}$} & $1 \mathrm{~A}$ & $\mathrm{VC}$ & 744,8 & 750,8 & 750,7 & 3 \\
\hline & $1 \mathrm{~B}$ & $\mathrm{CS}$ & 744,8 & 752,8 & 752,6 & 4 \\
\hline & $1 \mathrm{C}$ & $\mathrm{HF}$ & 744,8 & 764,8 & 764,3 & 11 \\
\hline & $1 \mathrm{D}$ & TOEP (1) & 744,8 & 750,8 & 750,7 & 3 \\
\hline & $1 \mathrm{H}^{1}$ & AR (1) & 739,7 & 747,7 & 747,5 & 8 \\
\hline \multirow{5}{*}{ FLOR } & $1 \mathrm{~A}^{1}$ & VC & 454,2 & 458,2 & 458,1 & 3 \\
\hline & $1 \mathrm{C}$ & $\mathrm{HF}$ & 454,2 & 472,2 & 471,7 & 12 \\
\hline & $1 \mathrm{D}$ & TOEP (1) & 454,2 & 458,2 & 458,1 & 3 \\
\hline & $1 \mathrm{E}$ & TOEP (2) & 454,2 & 460,2 & 460 & 3 \\
\hline & $1 \mathrm{H}$ & $\mathrm{AR}(1)$ & 454,2 & 460,2 & 460 & 3 \\
\hline
\end{tabular}

${ }^{1}$ Modelo seleccionado que minimiza AIC y BIC dentro de cada característica. REND: rendimiento de granos; CE: capacidad de expansión; AP: altura de planta; AE: altura de mazorca; FLOR: florecimiento masculino; REML: máxima verosimilitud restringida; VC: componentes de varianza; CS: simetría compuesta; HF: Huynh-Feldt; TOEP (1): diagonal 1; TOEP (2): diagonal 2; TOEP (3): diagonal 3; AR (1): auto-regresiva de orden 1.

puede afectar la rigurosidad de la investigación. Perri e Iemma (1999), analizando diferentes estructuras de covarianzas, encontraron que los valores del criterio de información de AIC pueden cambiar dentro de las estimativas de máxima verosimilitud restringida.

Los valores genéticos EBLUP del efecto de poblaciones para las características estudiadas se presentan en el Cuadro 6, considerando los modelos que minimizan los criterios de información de Akaike
(1974) y Bayesiano de Schwarz (1978), estructuras de $\mathrm{G}_{\mathrm{p}}$ (Cuadro 5). La clasificación de las poblaciones según su valor genético difirió entre las características. Para el rendimiento de granos, las poblaciones P5 y P4 mostraron valores genéticos positivos, mientras que para la capacidad de expansión estos mismos resultados incluyeron a las poblaciones P1 y P4. En tanto que P5 presentó, además de buen valor para el rendimiento, altos valores en altura de planta y altura de mazorca. Considerando que el rendimiento y la capacidad de ex- 
Cuadro 6. Predicción de valores genéticos del efecto de poblaciones vía mejor predicción lineal no sesgada empírica (EBLUP) por característica y considerando la estructura de covarianza seleccionada.

Table 6. Predicting breeding values of population effect by empirical best linear unbiased predictor (EBLUP) per trait and considering covariance structure selected.

\begin{tabular}{rrrrrrrrrr}
\hline \multicolumn{8}{c}{ Valores genéticos (EBLUP) } \\
\hline \multicolumn{2}{c}{ REND } & \multicolumn{2}{c}{ CE } & \multicolumn{2}{c}{ AP } & \multicolumn{1}{c}{ AE } & \multicolumn{2}{c}{ FLOR } \\
\multicolumn{2}{c}{ TOEP (3) } & \multicolumn{2}{c}{ TOEP (2) } & \multicolumn{2}{c}{ TOEP (2) } & \multicolumn{1}{c}{ AR (1) } & \multicolumn{2}{c}{ VC } \\
\hline P5 & 315 & P1 & 0,8 & P5 & 14,4 & P7 & 13,4 & P3 & 1,7 \\
P4 & 175 & P4 & 0,7 & P7 & 10,1 & P5 & 6,8 & P6 & 1,6 \\
P7 & -61 & P2 & $-0,4$ & P1 & 5,5 & P1 & 3,6 & P7 & 1,5 \\
P1 & -115 & P5 & $-0,9$ & P2 & 2,7 & P3 & 0,3 & P2 & 0,2 \\
P6 & -174 & P7 & $-1,0$ & P4 & 1,3 & P2 & $-0,7$ & P1 & $-0,1$ \\
P3 & -731 & P3 & $-1,7$ & P3 & $-5,8$ & P4 & $-3,0$ & P5 & $-0,4$ \\
P2 & -1.096 & P6 & $-3,7$ & P6 & $-24,2$ & P6 & $-12,4$ & P4 & $-4,4$ \\
\hline
\end{tabular}

REND: rendimiento de granos; CE: capacidad de expansión; AP: altura de planta; AE: altura de mazorca; FLOR: florecimiento masculino; TOEP (2): diagonal 2; TOEP (3): diagonal 3; AR (1): auto-regresiva de orden 1.

pansión representan características de interés económico y comercial, la población P4 puede ser considerada promisoria en su valor genético promedio para ambas características, cuando es comparada con otros orígenes. Quintana (2002) sostiene que la capacidad de expansión es el término utilizado en la evaluación del valor de una variedad o población de maíz para rosetas, o en este contexto, cuanto mayor es la capacidad de expansión, mayor será su valor comercial, ya que tal característica está asociada con la suavidad de la roseta.

Los coeficientes de correlación de Spearman, calculados entre los ranking de cada característica, se presentan en el Cuadro 7. La mayoría de las correlaciones de Spearman fueron no significativas ( $p>$ $0,05)$, cuyos valores variaron de $-0,79$ a 0,86 . El rendimiento y la capacidad de expansión solo se correlacionaron significativamente con el florecimiento masculino, pero ambos coeficientes fueron negativos. Las correlaciones de Spearman fueron no significativas $(p>0,05)$ entre el rendimiento y la capacidad de expansión. Esto quiere decir que la selección realizada únicamente para el rendimien- to de granos, tendría un efecto no significativo sobre la capacidad de expansión. Estudios de Sawazaki (1995) y Coimbra et al. (2001) confirman que la productividad de granos está inversamente correlacionada con la capacidad de expansión, dificultando la ganancia por selección simultánea cuando se refiere a las dos características en el mejoramiento poblacional (Miranda et al., 2003). Del mismo modo, Mora y Arnhold (2006) estimaron un coeficiente de correlación de Spearman no significativo calculado entre los ranking de ambas características obtenidos a través de BLUP, en familias $\mathrm{S}_{3}$ de maíz. La obtención de respuestas positivamente correlacionadas entre producción de granos y capacidad de expansión puede ser obtenida a través de determinados procesos de selección simultánea entre ambas características (Arnhold et al., 2006). En el presente estudio, de acuerdo a los valores genéticos obtenidos, la capacidad específica de combinación entre las poblaciones P4 y P5 debería ser investigada para la obtención de respuestas correlacionadas entre las características de capacidad de expansión y rendimiento de los granos.

Cuadro 7. Coeficientes de correlación de Spearman calculados entre las características agronómicas.

Table 7. Spearman rank correlation coefficients among the agronomic traits.

\begin{tabular}{lllll}
\hline Características & EXP & AP & AE & FLOR \\
\hline REND & $0,25 \mathrm{~ns}$ & $0,57 \mathrm{~ns}$ & $0,39 \mathrm{~ns}$ & $-0,68 *$ \\
EXP & & $0,43 \mathrm{~ns}$ & $0,14 \mathrm{~ns}$ & $-0,79 *$ \\
AP & & & $0,86 *$ & $-0,50 \mathrm{~ns}$ \\
AE & & & & $-0,07 \mathrm{~ns}$ \\
\hline
\end{tabular}

REND: rendimiento de granos; CE: capacidad de expansión; AP: altura de planta; AE: altura de mazorca; FLOR: florecimiento masculino. ns: Correlación de Spearman no fue significativo de acuerdo $(\mathrm{p}>0,05)$. *: Correlación de Spearman fue significativo $(\mathrm{p}<0,05)$. 


\section{CONCLUSIONES}

Los genotipos estudiados presentaron variabilidad significativa en todas las características agronómicas, indicando que es posible utilizar estas poblaciones para el avance de un programa de mejoramiento o de cruzamientos controlados.

Las estructuras de las matrices intergenotípicas dependieron de la característica evaluada, y sólo para el florecimiento masculino sería tipo com- ponentes de varianza, usualmente utilizado en el mejoramiento vegetal para la predicción de valores genéticos y estimación de parámetros de covarianza.

Las poblaciones P4 y P5 evidenciaron ser promisorias al considerar su valor genético para el rendimiento de granos, y las poblaciones P1 y P4 para la capacidad de expansión. Estas poblaciones pueden ser consideradas útiles para este programa de mejoramiento de maíz.

\section{LITERATURA CITADA}

Akaike, H. 1974. A new look at the statistical model identification. Trans. Autom. Control 19:716-723.

Arnhold, E., F. Mora, y A. Deitos. 2006. Correlaciones genéticas en familias $\mathrm{S}_{4}$ de maíz (Zea mays). Cien. Inv. Agr. 33:105-110.

Bard, Y. 1974. Nonlinear parameter estimation. 341 p. Academic Press, New York, USA.

Coimbra, R.R., G.V. Miranda, J.M.S. Viana, and C.D. Cruz. 2001. Correlações entre caracteres na população de milho-pipoca DFT-1 Ribeirão. Revista Ceres 48(278):427-435.

Dofing, S.M., N. D'croz-Mason, and M.A. ThomasCompton. 1991. Inheritance of expansion volume and yield in two popcorn $\mathrm{x}$ dent corn crosses. Crop Sci. 31:715-718.

García, M.V., M.J. Arturi, y O.E. Ansín. 2002. Variabilidad fenotípica y genética en poblaciones de pasto miel (Paspalum dilatatum Poir.). Agric. Téc. (Chile) 62:237-244.

Henderson, C.R. 1949. Estimation of changes in herd environment. J. Dairy Sci. 32:706-711.

Henderson, C.R. 1950. Estimation of genetic parameters. Ann. Math. Stat. 21:309.

Jahn, E., A. Vidal, y P. Soto. 2000. Sistema de producción de leche basado en alfalfa (Medicago sativa) y maíz (Zea mays) para la zona centro sur. II. Consumo y calidad del forraje. Agric. Téc. (Chile) 60:99-111.

Littell, R.C., G.A. Milliken, W.W. Stroup, and R.D. Wolfinger. 1996. SAS system for mixed models. 633 p. SAS Institute, Cary, North Carolina, USA.

Miranda, G.V., R.R. Coimbra, C.L. Godoy, L.V. Souza, L.J.M. Guimarães, and A.V. Melo. 2003. Potential to breeding and genetic divergence in popcorn cultivars. Pesq. Agropec. Bras. 38:681-688.

Mora, F., y E. Arnhold. 2006. Inferencia Bayesiana y metodología de modelos lineales mixtos aplicados al mejoramiento del maíz. Cien. Inv. Agr. 33:217-223.

Mora, F., O. Pupim-Junior, y C.A. Scapim. 2007. Predicción del efecto de cultivares de algodón en la presencia de interacción genotipo-ambiente. Cien. Inv. Agr. 34:11-19.
Perri, S.H.V., and A.F. Iemma. 1999. Procedimento MIXED do SAS ${ }^{\circledR}$ para análise de modelos mistos. Sci. Agric. 56:959-967.

Pflüger, L.A., and A.R. Schlatter. 1996. Isozyme variation in some races of maize from Argentina. Gen. Res. Crop Evol. 43:357-362.

Piepho, H.P., and J. Möhring. 2006. Selection in cultivar trials-Is it ignorable?. Crop Sci. 46:192-201.

Quintana, S.J.M. 2002. Análise dialélica em populações de milho-pipoca da raça Avati Pichinga (Zea mays L.). 63 p. Tesis de maestría. Universidad Estadual de Maringá, Maringá, Paraná, Brasil.

Reis, A.J.S., L.J. Chaves, J.B. Duarte, and E.M. Brasil. 2005. Prediction of hybrid means from a partial circulant diallel table using the ordinary least square and the mixed model methods. Genet. Mol. Biol. 28:314-320.

Rojas, C.G., y M.B. Manríquez. 2001. Comparación de ensilaje de trigo y de maíz en la engorda invernal de novillos. Agric. Téc. (Chile) 61:444-451.

SAS Institute. 1996. Statistical analysis system: user's guide. 956 p. SAS Institute, Cary, North Carolina, USA.

Sawazaki, E. 1995. Melhoramento do milho-pipoca. 21 p. Instituto Agronômico de Campinas, Campinas, São Paulo, Brasil.

Sawazaki, E., G.F.L. Morais, e A.A. Lago. 1986. Influência do tamanho e umidade do grão na expansão da pipoca South American Mushroom. Bragantia 45:363-370.

Scapim, C.A., C.A.P. Pacheco, A. Tonet, A.L. Braccini, and R.J.B. Pinto. 2002. Diallel analyses and heterosis in popcorn varieties. Bragantia 61:219-230.

Schwarz, G. 1978. Estimating the dimension of a model. Ann. Stat. 6:461-464.

Sokal, R.R., and F.J. Rohlf. 2003. Biometry: the principles and practice of statistics in biological research. $3^{\text {rd }} e d$. 850 p. Freeman and Company, New York, USA. 